\title{
APPLICABILITY OF PROCEDURAL VARIANTS IN ULTRASONIC TESTING
}

\author{
Gyula BAGYINSZKI, ${ }^{1}$ Enikő BITAY ${ }^{2}$ \\ ${ }^{1}$ Óbuda University, Donát Bánki Faculty of Mechanical and Safety Engineering, Budapest, Hungary, \\ bagyinszki.gyula@bgk.uni-obuda.hu \\ ${ }^{2}$ Sapientia Hungarian University of Transylvania, Faculty of Technical and Human Sciences, \\ Department of Mechanical Engineering, Târgu Mureș, Romania, ebitay@ms.sapientia.ro
}

\begin{abstract}
Ultrasonic testing (UT) is essentially the transmission of high-frequency $(0.25 \ldots 20 \mathrm{MHz}$ ) sound waves (mechanical vibration and energy) into a material, in order to interact with the macro-structural constituents of the material which reflect and/or weaken it. Ultrasonic testing - as one of the most common nondestructive material testing (NDT) methods - thanks to its many variants, is able to operate in a mobilized and highly automated fashion; it can be performed on most material types; high accuracy and reproducibility can be achieved in error detection, and it is generally enough if only one side is accessible; changes in elevation can also be verified with this method besides distance measurement, and it is also suitable for determining certain material characteristics. The present article reviews the application technology characteristic of the procedural variants.
\end{abstract}

Keywords: mechanical vibration, coupling, transmitter, receiver, discontinuity, fault signal.

\section{Introduction}

The energy supplied by the electricity network's $50 \mathrm{~Hz}$ electromagnetic vibration (alternating voltage and current) is converted to high-frequency mechanical vibration (ultrasound) and energy by a resonator which is based on the phenomenon of positive or negative longitudinal magnetostriction. It is a form of demonstration of the relation between the magnetisation and mechanical state of stress of ferromagnetic bodies; or electrostriction, as the reciprocal piezoelectric effect [1].

When applying magnetostriction, the axial alternating magnetic field strength created by converting the electrical network's low frequency to high frequency and feeding it into a coil is demonstrated as mechanical vibration (low-amplitude longitudinal elastic deformation) in the ferromagnetic material practically placed into the magnetic field. Therefore the ferromagnetic (iron) core of the coil fed with alternating current performs powerful longitudinal vibrations, i.e. emits ultrasound waves, provided its natural frequency equals the frequency of the alternating current.

Electrostriction is a particularity of active dielectrics, which is an alternating elastic deformation caused by an alternating electric field (voltage), i.e. mechanical vibration, or ultrasound in case of an adequately high frequency. Due to resonance, the amplitude of this mechanical vibration is highest when the frequency of the vibrations of the electric field equals one of the natural frequencies of the dielectric crystal. The inverse phenomenon of this is piezoelectricity, when elastic deformation (e.g. reflected ultrasound) causes charges (electric voltage) of opposite polarity to appear on the surfaces of the appropriately shaped crystals of the active dielectric, which may be evaluated as an amplified signal.

In the course of ultrasonic testing, a quartz crystal changes its dimensions periodically due to electrostriction, generating pressure waves (ultrasound) in the surrounding medium ("transmitter" mode). As the inverse of the phenomenon, if the crystal is subjected to mechanical load (e.g. 
reflected ultrasound), a voltage proportional to the frequency of the vibration is generated in it (as a signal that can be evaluated (in "reception" mode).

Mechanical waves propagate in a number of ways in the sound space: by longitudinal (pressure wave), transverse (shear wave), and Rayleigh (surface) waves. For longitudinal waves, the movement of particles and the direction of propagation are identical, while they are perpendicular for transverse waves. For Rayleigh waves, the particle movement characteristic of the transverse wave is only applicable to surface layers; the waves become increasingly weaker with depth beneath the surface.

If discontinuities can be found in the sound path, part of the mechanical energy will be reflected from the discontinuity, as from reflecting surfaces. The reflected sound wave's signal is then converted back into an electrical signal, and its intensity is shown on a suitable display. The penetration time of the sound waves can be directly related to the distance travelled by the signal. Based on the signal it is possible to determine the information concerning the reflector's location, size, orientation and other characteristics. Depending on the purpose of the application, a number of ultrasound test procedure variants are distinguished.

\section{Sound-Optical (SO) procedure}

Its principle is similar to that of X-ray inspection (Figure 1a), as the ultrasound is reflected from the fault in the tested object, but the waves propagating through the faultless areas are detected, converted to optical signals and the presence of discontinuities is thus established. A sketch of the procedure is shown in Figure 1b. [2].

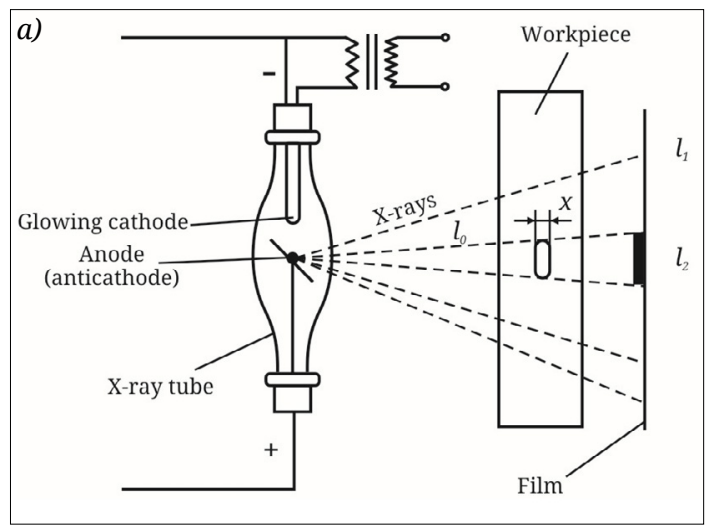

\section{Through transmission (TT) procedure}

This procedure uses separate transmitters to emit and receive ultrasound. The transmitter probe must be attached to one side of the test sample with the reception transducer attached to the opposite side. As the sound passes through the test piece and a discontinuity blocks the propagation of the wave, part of the ultrasound radiation will not be able to propagate further (the signal will weaken), therefore the error signal appears as a reduction of the intensity of the end signal. The receiver placed opposite the transmitter detects the transmitted ultrasound and returns an end signal of proportionately lower energy at the location of faults. The fault signal is given by the difference of the end signals of the perfect material and the material containing the fault (Figure 2a) [2]. The procedure allows displaying the shape and extent of the faults, but it does not show its depth and type.

\section{The pulse echo (PE) procedure, with perpendicular or angle head}

In this procedure, the sound beam is driven into the tested object via a couplant, which is then reflected from the "reflectors" blocking the path of propagation. The reflected mechanical vibration induces an electrical signal in the receiver, which can be displayed in the cathode ray tube of an analogue oscilloscope or the display of a digital oscilloscope. In the case of a discontinuity, the reflected signal returns faster than those reflected from the back wall, therefore the distance of the error signal appearing on the screen from the inbound signal is proportional to the depth measured from the surface (Figure 2b) [2]. If the $c$ sound velocity in the material is known, the fixed time interval

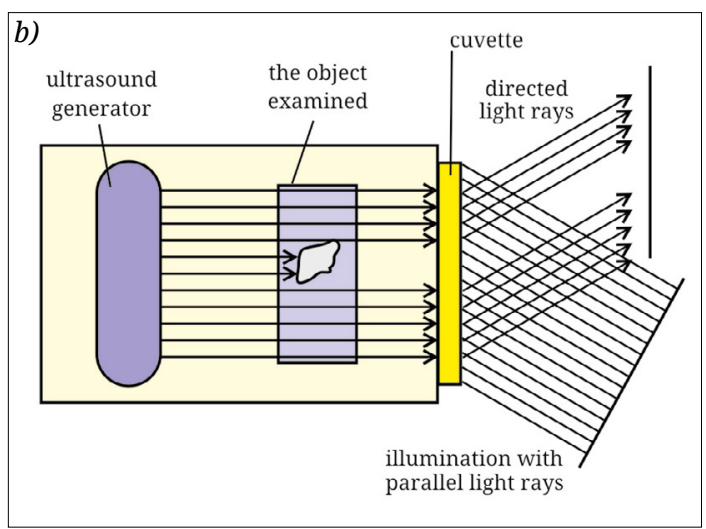

Figure 1. Sketch of the X-ray examination and the ultrasound phonetical and optical procedure 

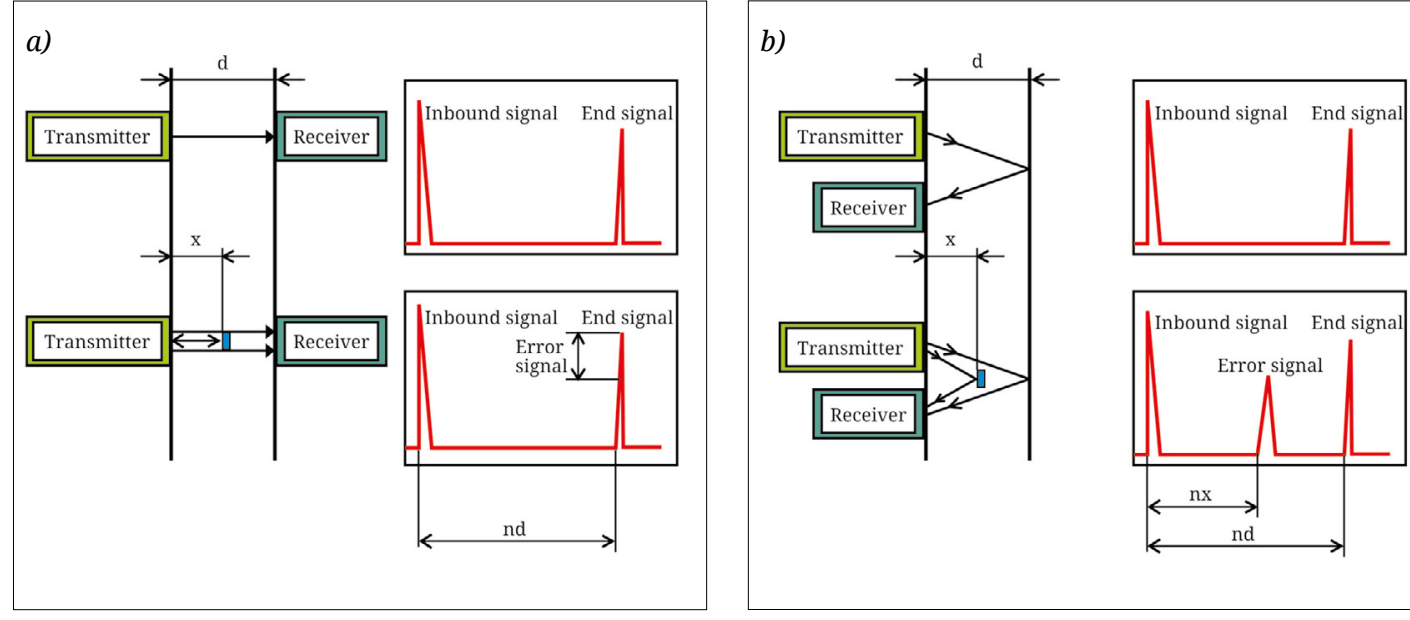

Figure 2. The principle of through transmission and pulse echo procedures

$(\Delta t)$ is used to determine the distance (x) travelled in the material:

$$
x=c \cdot \Delta t \text {. }
$$

From the shape and size of the fault signal, the type and extent of the discontinuity can be deduced.

Judgment of the type of discontinuity based on the signal fed back from the fault can be difficult at times, requiring well trained and experienced examination personnel. E.g. a pin-type signal "flashing" at particular positions comes in upon moving the inspection head from a spherical gas inclusion and a "stepped and spiked" signal from a solid inclusion having multiple reflective surface elements.

The difference between the shapes of discontinuities can be better evaluated by applying two irradiation directions. The reflective surface of the volumetric spherical fault results in the same signal, regardless of direction, but for a surface fault, the component of the signal in the direction of radiation is reduced, due to the reflection of the radiation opposite to its angle of incidence, thereby reducing the height of the signal.

In the course of the ultrasonic tests, it may occur that fault signal-like images are obtained from areas where there are no discontinuities at all. Reasons of false fault signals may be:

-interference due to faulty arrangement of electric components;

- cracked transmitter head, interfering with the emitted signal;

- air bubbles in the couplant;

- waves reflected from various edges, i.e. dependency on the shape of the tested piece;
- granule boundaries;

- change of the waveform;

- waves reflected from the crown and the root as well as the heat affected zone in the case of welded joints.

\section{Time of Flight Diffraction (ToFD) pro- cedure}

Diffraction is the change in the sound's wavelength when interacting with a discontinuity. This phenomenon may be applied in situations where no real reflection can be obtained, but sufficient diffraction occurs to change the propagation time of the sound at a particular level of sound pitch. This procedure is applied to detect fault peaks placed perpendicularly to the contact surface of the probe, and to examine the opposite wall, e.g. to detect corrosion [3].

In time of flight diffraction systems probes placed at the opposite side of the examined area are also used. The transmitter probe emits an ultrasound impulse, which is received by the receiver probe on the other side. In the intact part, the signals picked up by the receiving probe originate from two waves: one propagates along the surface (lateral wave), while the other is reflected from the distant wall (back wall reflection). If discontinuities such as cracks are detected, the diffraction of the ultrasound wave will take place between the two peaks (e.g. the lower and upper peaks) of the crack (Figure 3). With the help of the measured propagation time of the impulse, the depth of the crack peaks is automatically calculated by trigonometry. 


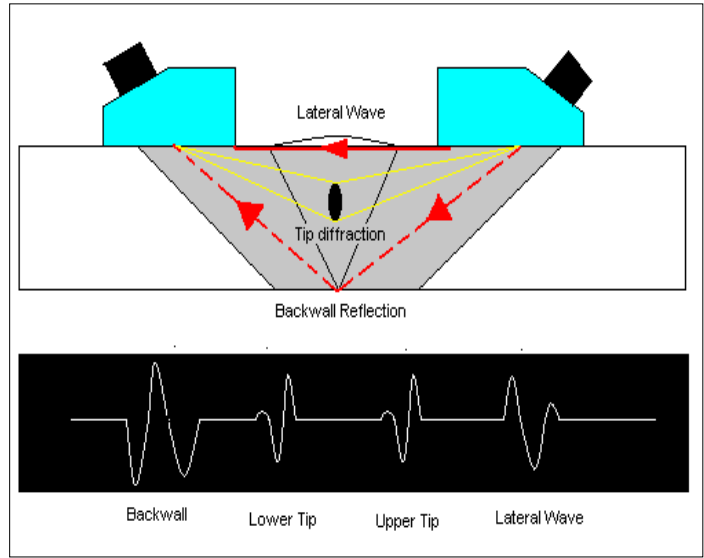

Figure 3. The principle of the time of flight diffraction procedure

The time of flight diffraction procedure offers a high degree of accuracy for measuring the size of crack-like faults in critical walls. Generally, an accuracy greater than $\pm 1 \mathrm{~mm}$ can be achieved in a broad thickness range of materials, from which pressurised structures are built, for instance.

Advantages:

- High speed of detecting discontinuities.

- Precise positioning and measurement of discontinuities.

- The proven reproducibility and accuracy is $0.5 \mathrm{~mm}$.

- Continuous digital imagery with graphical images.

- Detection is independent of the type and direction of discontinuities.

- Consistent (uniform) results before and after heat treatment.

- Consistent results in the course of preliminary and in-process control.

-Precise procedure to measure the growth of the discontinuity.

-Ensures a higher probability of detection.

- Planar discontinuities may be demonstrated which are not perpendicular to the tested surfaces.

- Risk reduction is improved.

- There is no need to evacuate the surrounding areas while working, as the procedure uses no harmful radiation.

- The inspection results are immediately available.

- The test may be conducted above $200^{\circ} \mathrm{C}$.

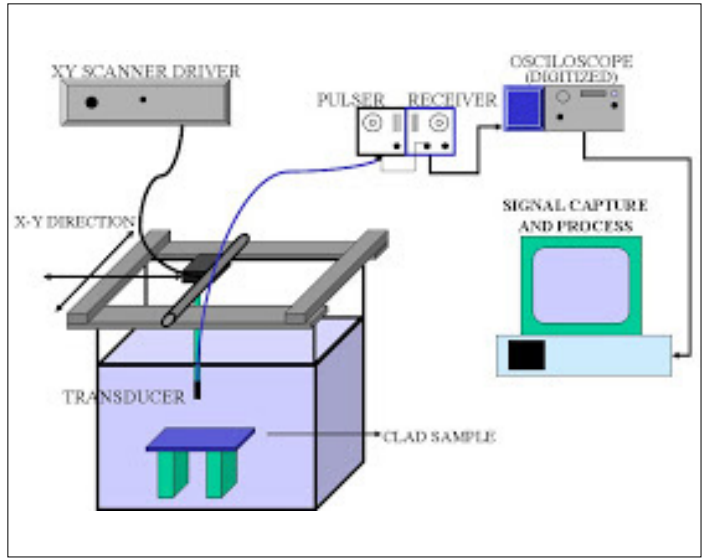

Figure 4. Sketch of the immersion procedure

\section{Immersion procedure}

In the case of large workpieces or those of complex shape, the proper coupling of the ultrasound probe and the surface of the test piece may be problematic. For reasons of "convenience" these items are generally immersed in a water tank. The ultrasound probe on the surface of the tested object placed in the tank filled with water is moved, while the vibration enters from the water into the tested area. After the sound interacts with the internal material structure and the occasional faults or the opposite surface of the product, the reflected sound wave returns to the probe (Figure 4.). The arrival time and amplitude of the echo permits identifying where and from what the signal is originating.

The ultrasound probe is often fitted to a robotic arm with translational and rotational movements. The scanning coverage of the product may then be automated by using pre-programmed paths, allowing several passes in a particular area, i.e. collecting data by scanning, whose images can be viewed.

Such use of water as a couplant eliminates variations in the quality of coupling that may emerge during a conventional contact test. In addition, checking rougher surfaces also becomes possible without loss of coupling.

Immersion ultrasonic testing is highly versatile and can be configured well to single-probe, phase-controlled probe and other advanced techniques. High frequency sensors are used in narrower material thickness ranges and in easily radiated materials, such as unalloyed steel, while lower frequencies are needed for thick profiles 
and materials of greater attenuation, e.g. stainless steels and plastics.

The typical verification tasks conducted by applying this test procedure include mapping of the corrosion behaviour of steel plates and pipes, verification of the welding seams of technological pipes, quality control of automobile parts and the analysis of composite damages.

Advantages:

-Efficient inspection of large components with high resolution, contrary to the conventional ultrasound procedure.

- The diversity of the shape, size and material of the inspected components.

- High spatial precision of scanning, ensured by automated probe movement and data collection programmes set in advance.

- High rate of reproducibility due to constancy of water as a couplant.

- Continuous record of inspection with the corresponding images scanned..

Limitations:

- Not suitable for in-process controls due to the necessary immersion tank.

- The components must be immersed in water, which may be prone to corrosion, depending on the material type.

- The access limitations of the probe may restrict controlling complex geometries.

\section{Air Coupled (AC) procedure}

Certain tests and materials do not tolerate the use of wet coupling, therefore under certain circumstances, air couples, non-contact ultrasonic testing (Figure 5) may also be performed. This is therefore the application of sound via an air gap, usually by applying lower frequencies [3].

Depending on the material and/or technology, some materials swell, peel, corrode or are damaged in other ways. The air coupled technology allows inspection of such highly attenuating materials that may only be tested with little or no success using water-coupled ultrasonic testing. These include among others composites, honey cell structured materials, foam, ceramics, wood and concrete.

\section{Electromagnetic Acoustic Transducer Testing (EMAT)}

This is a contact free test procedure, using electromagnetic sound generation and reception without direct contact or wet coupling with the component. Electromagnetic acoustic transducer testing is particularly useful if the environment is excessively hot, cold, clean or dry. As in the case of conventional ultrasonic testing, electromagnetic acoustic transducer testing also allows the use of radiation perpendicular or at an angle to the material [4].

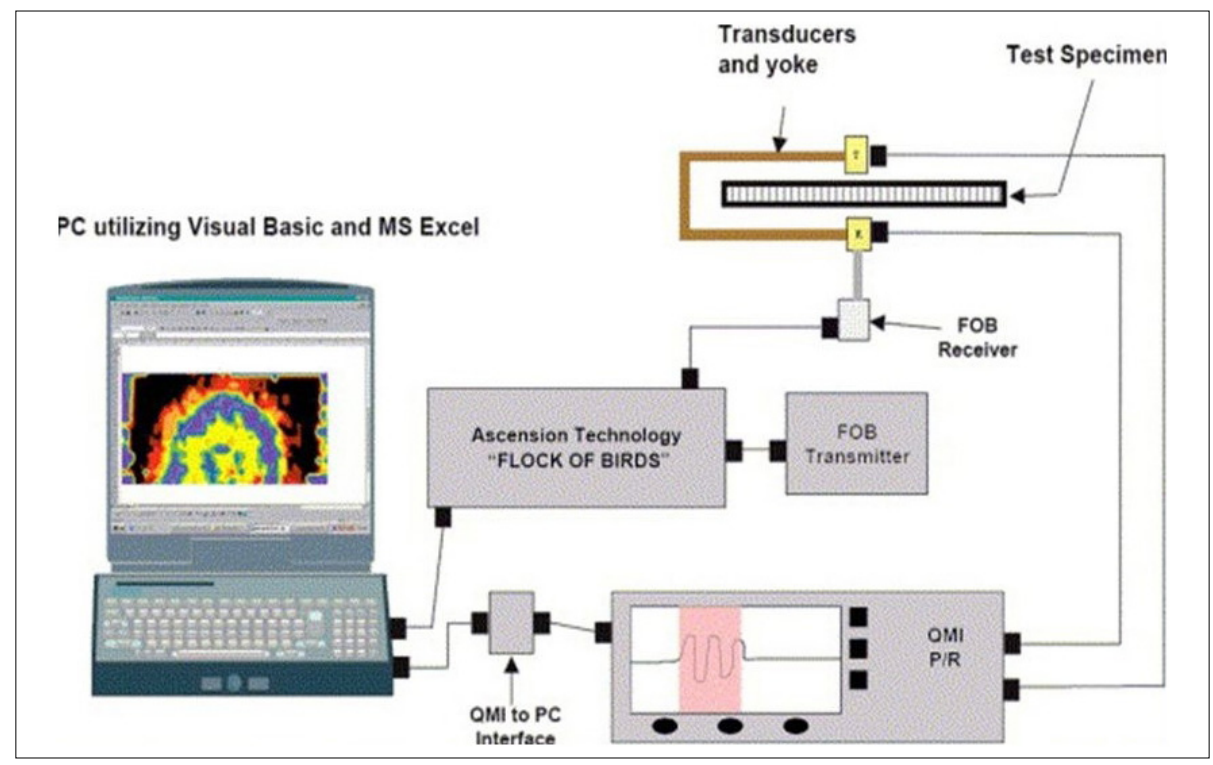

Figure 5. Sketch of the air coupled procedure. 


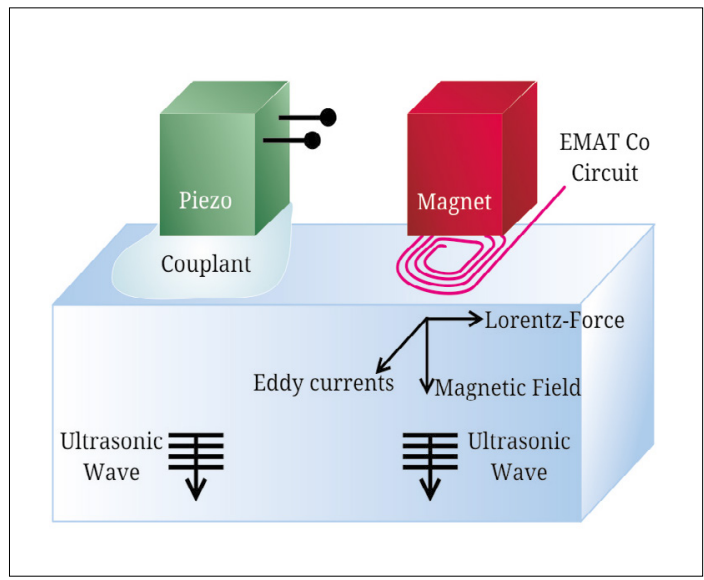

Figure 6. The principles of conventional and electromagnetic acoustic transducer testing.

Electromagnetic acoustic transducer testing (Figure 6) is an ultrasonic testing technique that generates the signal in the controlled part. The procedure generates ultrasound waves in the test object, with the help of two interacting magnetic fields. The field of relatively high frequency generated by the electric coil(s) interacts with the low-frequency or static field of the strong magnet(s), to generate a Lorentz-force.

This "interference" is then transmitted to the crystal lattice structure of the material, generating a flexible wave. In the inverse process, the interaction of flexible waves generates a current in the reception coil in the presence of a magnetic field. In ferromagnetic materials, magnetostriction generates further stresses that elevate the signals to far higher levels than the Lorentz-force is capable of on its own. Various waves may be generated using different combinations of high frequency coils and magnets.

As the sound is generated in the tested part and not the transducer, the electromagnetic acoustic conversion testing has the following advantages over the use of conventional piezoelectric converters:

-It is a dry test, i.e. the procedure requires no couplant to convey the sound, so it is highly suitable to test very hot and very cold parts, integrated in an automated environment.

- The procedure may be used for inspection through layers of coating and is furthermore not affected by the presence of contaminants, oxidation or roughness.

- The sensor is easy to install and its installation angle does not affect the direction of propagation.
- The only practical device is the generation of shear waves with the help of horizontal polarisation, without exerting large mechanical pressure of low-density connections, which may hinder scanning the component.

- The combination of the coil-antenna type structure of the procedure and a multiple-cycle excitation ensures a high degree of specification in the frequency range, enabling selection of the precise wave mode, which is of great significance from the perspective of directed wave generation and interpretation.

The advantage of automation is the integration of sensors into commercially available and collaborative robots. The customized software for data collection and display, which may be further tailored to suit individual needs, offers an interference-free and intuitive user experience.

\section{Phased Array Ultrasonic Testing (PAUT)}

In the case of limited accessibility, a fault finding procedure is required that can indicate faults with adequate certainty over the entire cross section. Phased array ultrasonic testing is the most suitable procedure in such cases. It allows varying the ultrasound radiation angles and dynamic depth focusing. The phase-controlled radiation provides recordable digital imagery. Figure 7a. compares conventional and phased array ultrasonic testing $[5,6]$.

In the case of the phase-controlled test procedure, the inspection head is made up of several resonators (piezoelectric crystals), which can function independently from one another. With these resonators, a wavefront is generated whose angle of incidence can be varied (Figure 7b):

$$
\Theta=\arcsin (C \cdot \Delta t / w) \text {, }
$$

where $C$ is the phase speed in the material, $\Delta t$ is the phase delay of the impulses of the particular probes and $w$ is the distance of the probe's centerline.

As a wider range is examined here, it is not necessary, for example, to perform scanning movements with the head when inspecting weld seams as in a conventional test; it is sufficient to guide it parallel to the seam. A significant advantage of this is that the simpler movement helps in recording the test, which is very useful for maintenance, state assessment and even state tracking.

As the inspection head is made up of a number of resonators and upon sectoral tests, they use one angle range (e.g.: $30 . . .55^{\circ}$ ), the probability of 

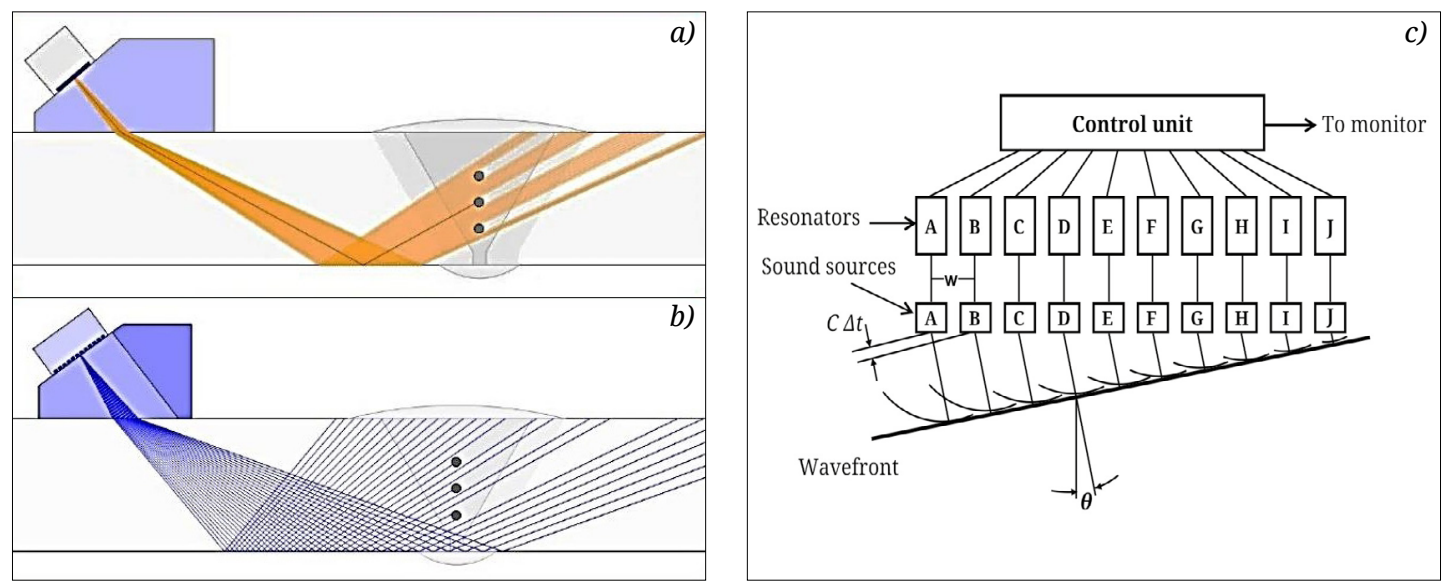

Figure 7. Comparison of conventional and phased array ultrasonic testing

demonstrability is increased, thereby allowing access to much more information, and discontinuities beneath one another can also be better demonstrated. The precise size of faults and their positions can also be displayed in 3D.

\section{Full Matrix Capture (FMC)}

FMC is the advanced development of the PAUT, using the same probes. Its main advantage is that there is no need to focus or direct the sound beam, as the entire testing area is within the field of focus (Figure 8). It is relatively tolerant of various defects and structural noise. This greatly simplifies adjustments and use. Its disadvantage is that the size of the files generated is very large and data collection speed may be slower than in the case of PAUT [4].
Full matrix capture (FMC) is a data collection strategy that allows recording of all possible transmission and reception combinations. The collected data are processed in real time with the optimised version of the algorithm, which generates fully focused images from the FMC data. The objective of the new technique is increasing the reliability of ultrasonic testing at reduced costs and greater certainty.

\section{Virtual Source Aperture (VSA)}

The VSA is an FMC-variant that retains most of the advantages of excellent image quality, but has a significantly reduced file size and more favourable data collection speed, effectively surpassing PAUT. The positioning of the virtual source - at a certain distance behind the transducers - allows

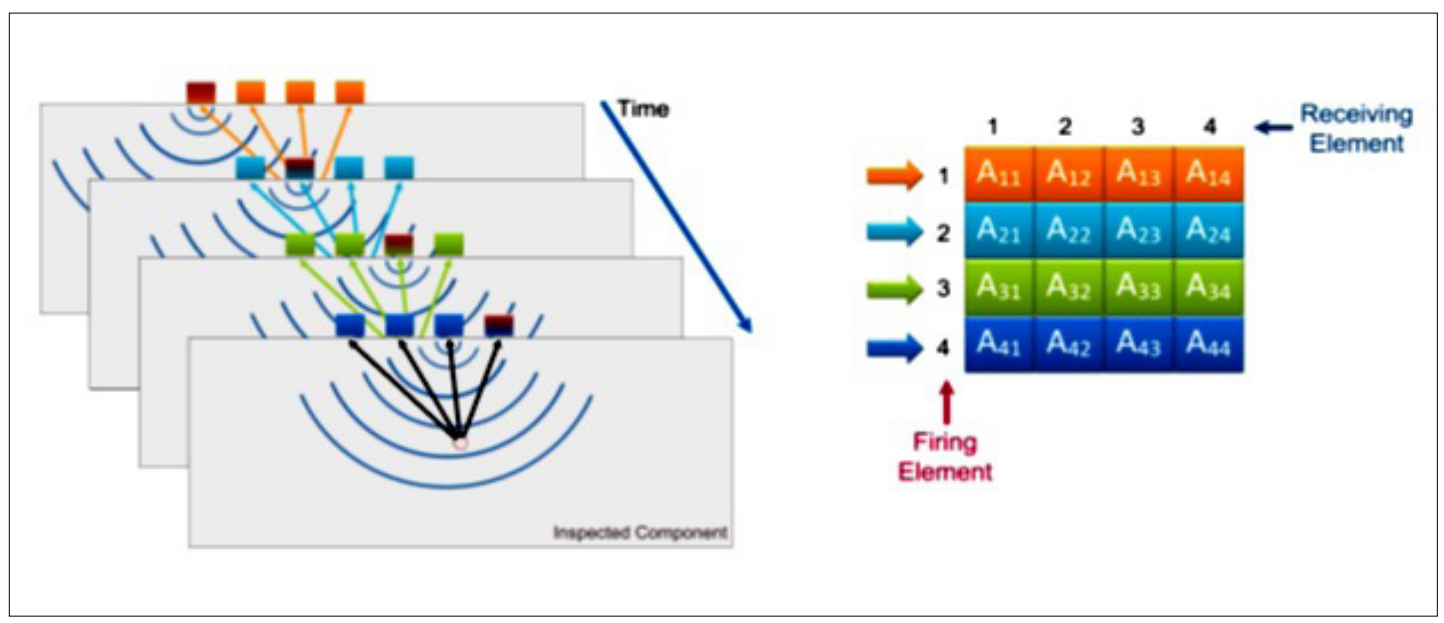




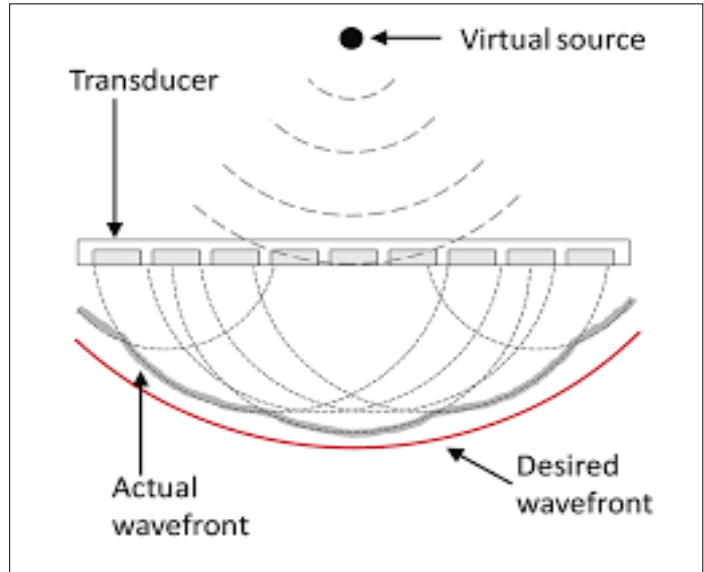

Figure 9. Sketch of the virtual source aperture procedure.

multiple elements to radiate, simulating ultrasonic transmission according to the law of delay. Figure 9 shows that the ultrasonic energy is directly emitted by the virtual source, generating spherical waves in the form of unfocused and highly divergent radiation [4].

\section{The resonance procedure}

A resonance condition emerges when the thickness of the material equals the wavelength of the sound or half of any multiple thereof. The wavelength regulation of the ultrasound is achieved by regulating its frequency. If a variable-frequency transmitter is available, it is advisable to adjust it so as to create the resonance condition for the thickness of the plate tested. The resonance condition is easily recognised by the increase of the impulse received.

The procedure, based on the principle of resonance is used to determine the thicknesses of thin plates. A plate of thickness $d$ is brought into a resonance condition with the help of ultrasound with propagation speed $c$. The resonance frequency

$$
\text { frez }=c / 2 d \text {, }
$$

from which the thickness can be determined.

\section{Automated and partly automated tests}

Automated systems are used where large quantities of similar components must be inspected. These systems operate with one or more probes, which the control unit places on the test piece, moving them according to a predefined scanning scheme. The ultrasonic signals are processed by

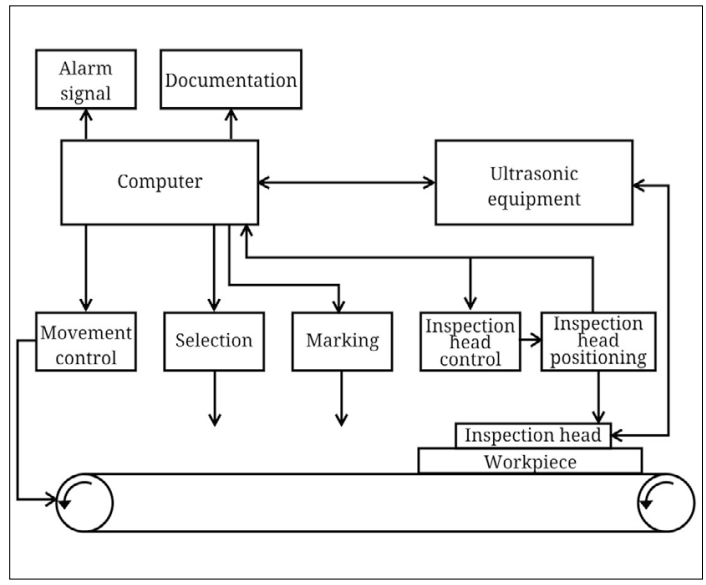

Figure 10. Sketch of an automated ultrasonic testing system.

the evaluation unit, and displays them on the monitor where possible. All the measured data and information regarding the probe's position are fed into a computer where they are further processed and evaluated. The computer also controls the marking and selecting tool as well, which indicates the faults on the objects tested. A further task of the computer is to control the movement of the workpiece, and to indicate the various testing conditions. Figure 10. shows the block diagram of a typical automated ultrasonic testing system [6].

\section{Conclusions}

Ultrasonic testing is in widespread practical use as a means of post-production product quality testing, or in the course of operation, to detect faults arising from the operation. The reliability of ultrasonic testing is increasing with the development of digital technology. Further advantages of these procedures can be encountered in the areas of storage, reproduction and transmission of the data.

\section{References}

[1] Bagyinszki Gy., Bitay E.: Ultrahangos hegesztés alkalmazástechnikai jellemzői. In: A XXIII. Fiatal műszakiak tudományos ülésszak előadásai. Proceedings of the $23^{\text {rd }}$ international scientific conference of youngth engineers, Kolozsvár/Cluj, Románia, Műszaki Tudományos Közlemények 9. (2018) 31-34.

https://doi.org/10.33894/mtk-2018.09.03

[2] Gál I.: Anyagvizsgálat előadásvázlat. Miskolci Egyetem, Mechanikai Technológiai Tanszék, 2007. 
[3] What is Non-Destructive Testing (NDT)? Methods and Definition

https://www.twi-global.com/technical-knowledge/faqs/what-is-non-destructive-testing\#MethodsofNonDestructiveTesting

[4] EMAT Technology.

https://www.innerspec.com/en-gb/knowledge/ emat-technology/
[5] Phased Array Services.

https://www.acuren.com/inspection/advancednde-ndt/phased-array/

[6] Training Guidelines in Non-Destructive Testing Techniques - Manual for Ultrasonic Testing at Level 2, International Atomic Energy Agency, Vienna, 2018. 